\title{
High-throughput fabrication of compact and flexible bilayer nanowire grid polarizers for deep-ultraviolet to infrared range
}

\author{
Li Wang, ${ }^{\text {a) }}$ Helmut Schift, Jens Gobrecht, and Yasin Ekinci \\ Laboratory for Micro- and Nanotechnology, Paul Scherrer Institute, 5232 Villigen PSI, Switzerland \\ Per Magnus Kristiansen \\ Institute of Polymer Nanotechnology, University of Applied Science and Arts Northwestern Switzerland \\ (FHNW), 5210 Windisch, Switzerland \\ Harun H. Solak \\ Eulitha AG, 5232 Villigen PSI, Switzerland \\ Konstantins Jefimovs \\ Laboratory for Electronics/Metrology/Reliability, EMPA, Swiss Federal Laboratories for Material Science \\ and Technology, Ueberlandstrasse 129, 8600 Dübendorf, Switzerland
}

(Received 4 January 2014; accepted 21 April 2014; published 30 April 2014)

\begin{abstract}
The authors present the design and fabrication of a bilayer metallic wire-grid polarizer with a period of $80 \mathrm{~nm}$ on a flexible polymeric substrate optimized for broadband operation ranging from the infrared down to the deep-ultraviolet range. Their high-throughput fabrication over large areas is realized by nanoimprint lithography by producing the imprint master stamps using extreme ultraviolet interference lithography. Optical measurements show that the fabricated bilayer polarizer covers a broad spectral range, starting from wavelength of $280 \mathrm{~nm}$. Transverse magnetic transmission of $70 \%$ and an extinction ratio of $30 \mathrm{~dB}$ were realized. (c) 2014 American Vacuum Society. [http://dx.doi.org/10.1116/1.4874318]
\end{abstract}

\section{INTRODUCTION}

Metallic wire-grid polarizers (WGPs) transmit TMpolarized light (transverse magnetic) and reflect TE polarization (transverse electric) and have been widely used in radio, microwave, and infrared (IR) spectra regions. ${ }^{1}$ They are compact, planar, and compatible with integrated circuit fabrication, simplifying their use as optical components in nanophotonics, fiber optics, displays, and detector devices. As a rule of thumb, the shortest operating wavelength for a WGP to work as an efficient polarizer is about three times larger than the period of the grid. The long wavelength operation is not limited in principle, but in practice is restricted by the absorption of the substrate. Today, commercially available WGPs have a period of about $150 \mathrm{~nm}$ due to nanofabrication challenges, which limit them to an effective operating wavelength down to about $450 \mathrm{~nm}$, and an extinction ratio of about 500 to 2000 in the visible (VIS) wavelength range. ${ }^{2,3}$ Since the availability and performance of polarizers are very limited for applications in the ultraviolet (UV) range, WGPs working in these wavelength regions offer a promising solution. On the other hand, WGPs with a spectral working range down to a wavelength of less than $300 \mathrm{~nm}$ require a period of about $100 \mathrm{~nm}$ or even less, which is technologically very challenging. Therefore, it is of high technological interest to establish feasible and cost-effective fabrication methods for highperformance WGPs operating at such short wavelengths.

Among various nanofabrication techniques that can be used to create grating structures with sub- $100 \mathrm{~nm}$ periodicity, nanoimprint lithography (NIL) is considered to be most suitable for high-throughput, large-area, and cost-effective

${ }^{a)}$ Electronic mail: li.wang@psi.ch patterning. ${ }^{1,4}$ Chen et al. demonstrated high-efficiency flexible metal WGP using NIL and shadow metal evaporation technique. ${ }^{5}$ Ahn et al. reported metal WGP fabricated by roll-to-roll NIL on a flexible plastic substrate. ${ }^{6}$ We note that all of the aforementioned WGPs work only in the visible spectral range.

In this paper, we report on the fabrication and performance of bilayer WGPs (Ref. 7) with a period of $80 \mathrm{~nm}$ on a flexible polymer substrate with a broadband operation range of deep-ultraviolet (DUV) to IR region. Bilayer WGPs are fabricated by nanostructuring polymer foils by NIL and subsequent metal deposition onto these patterns, leading to two metal gratings separated by a certain distance and laterally shifted by half a period on the flexible polymer foil [see Fig. 1(a)]. The high-resolution stamps were fabricated with extreme ultraviolet interference lithography (EUV-IL) and subsequent dry etching into silicon, followed by stamp copying using NIL. Since the fabrication process involves only polymer patterning and metal evaporation, avoiding dry etching or lift-off processes, which is challenging for sub$50 \mathrm{~nm}$ features, a bilayer WGP is simpler and less costly than a single-layer WGP. ${ }^{3}$ Bilayer polarizers are also advantageous for large area applications. For instance, in display applications, where very large area polarizers are needed, which can be achieved by roll-to-roll imprint lithography on flexible polymer substrates. In stark contrast, pattern transfer through etching and lift-off is very difficult to realize for large samples. Another advantage of bilayer WGPs is the defect tolerance. Since the last step of the fabrication process is metal deposition onto prepatterned polymer gratings, fabrication defects, such as pinholes, which reduce the polarization factor significantly, can be avoided. Bilayer WGPs also provide high extinction ratios, as their behavior, in a first 


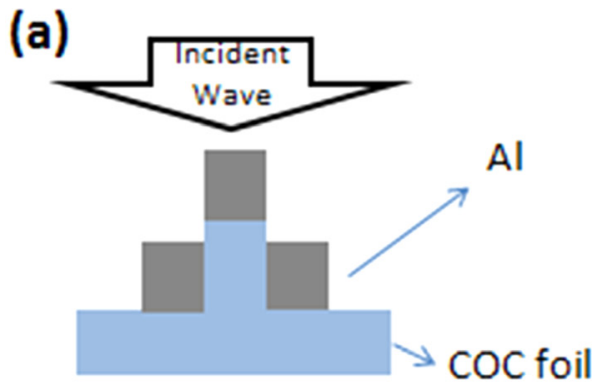

(b)

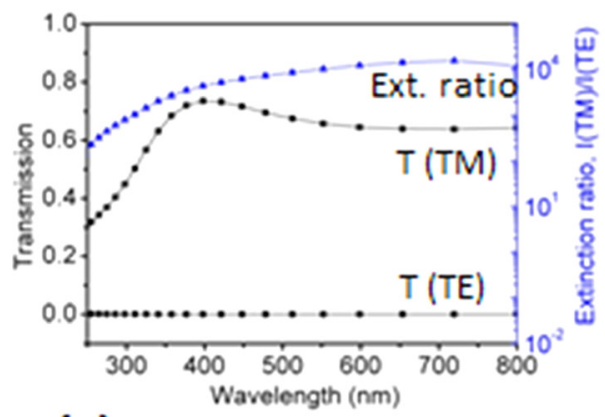

(c)

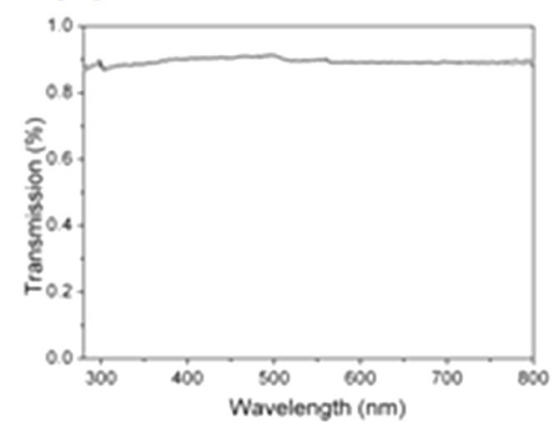

FIG. 1. (Color online) (a) Schematic illustration of a designed bilayer Al WGP. (b) Simulated optical performance results of a proposed bilayer Al WGP in the DUV to IR spectral regions on COC foil substrate. (c) Experimental measured transmission spectrum of a $130-\mu \mathrm{m}$-thick COC foil prepared by NIL.

approximation, is like the optical response of two singlelayer WGPs in tandem. ${ }^{7}$

Moreover, in this work, we introduce a new approach in the fabrication process flow in order to overcome the challenges in nanofabrication and to further reduce the fabrication costs. In order to circumvent the difficulty in writing dense and large-area patterns for NIL stamps by electron beam lithography (EBL), we introduce EUV-IL as an intermediate step for fabrication of stamps for NIL. ${ }^{8}$ Here, EBL is used for fabricating a grating for EUV-IL, which in turn is used for the fabrication of the NIL stamp originals. We used this EBL-written grating as a transmission grating for achromatic Talbot EUV lithography. ${ }^{9}$ This method provides multiple advantages. First, the patterns obtained by EUV-IL have half the period of the grating used. Second, EUV-IL allows high throughput fabrication, since it is a parallel writing process. A typical exposure of a single grating takes about $10 \mathrm{~s}$ so that multiple NIL stamps are exposed in very short time, enabling exhaustive optimization of NIL stamps or replacement of the stamps after extensive usage. In fact, we produced daughter stamps from the NIL stamp originals. Thus, we have realized WGPs with this four-stage lithography, i.e., EBL, EUV-IL, NIL for daughter stamps, and NIL for WGPs, which substantially reduces the costs at each stage. Optical characterization of the fabricated WGPs revealed that the fabricated bilayer polarizers based on cyclic olefin copolymer (COC) foil, which is an amorphous thermoplastic polymer, and used in a variety of applications including packaging films, lenses, vials, displays, and medical devices, display broadband polarization, starting from a wavelength of $280 \mathrm{~nm}$, with a TM transmission of $70 \%$, and an extinction ratio of $30 \mathrm{~dB}\left(10^{3}\right)$, demonstrating the high performance and broadband advantages of the bilayer WGPs.

\section{EXPERIMENTAL DESIGN, RESULTS, AND DISCUSSION}

Before fabrication of the WGPs, we performed extensive theoretical studies on their optical properties and optimized the performance by tuning the structural parameters within the range of parameters feasible for nanofabrication. The designed metallic bilayer WGP on patterned COC foil is illustrated in Fig. 1(a). Electromagnetic properties of the bilayer metallic wire-grids were investigated with numerical simulations in DUV to IR region by applying finite-element methods in the frequency domain using a commercial code (Comsol Multiphysics). ${ }^{10}$ The geometry of the simulation domain consisted of one unit cell of the periodic grating structure for a bilayer polarizer as shown in Fig. 1(a). Aluminum (Al) was chosen as the material due to its excellent optical properties in the UV range, process compatibility, oxidation resistance, and broad operating wavelength range. The performance of the polarizers, i.e., TM transmission and extinction ratio, specifically in the UV spectral region were maximized by optimization of the structural parameters, such as period, duty cycle (line-spacing ratio), spacing layer thickness (depth of patterned polymer grating), and metal thickness, within the range of parameters accessible with currently available nanofabrication techniques. Based on this analysis and optimization of the nanostructure parameters, a broadband bilayer WGP on a COC foil substrate was designed. COC was chosen due to its transparency in the wavelength range of interest and comparatively high heat resistance. Figure 1(c) shows a typical transmission spectrum of COC foil of $130 \mu \mathrm{m}$ thickness from the UV to the IR spectra region exhibiting $90 \%$ transmission.

The simulation results indicated that an average extinction ratio over $40 \mathrm{~dB}$ and transmission efficiency over $70 \%$ could be achieved down to $250 \mathrm{~nm}$ wavelength with a period of $80 \mathrm{~nm}$, a duty cycle of 0.5 , an $\mathrm{Al}$ thickness of $40 \mathrm{~nm}$, and a patterned polymer thickness of $60 \mathrm{~nm}$ on COC foils [see Fig. 1(b)]. As our extensive simulations showed, this kind of bilayer WGP also has a wide acceptance angle up to $60^{\circ}$ for $300 \mathrm{~nm}$ wavelength and $80^{\circ}$ for $700 \mathrm{~nm}$ wavelength. ${ }^{9}$

To realize a cost-effective fabrication of the designed bilayer WGP, NIL was selected among various nanofabrication techniques. One of the key issues to ensure a successful 
nanoimprint process is the master stamp, whose quality determines the final quality of the patterned polymer grating on a foil. With current EBL tools, generation of dense arrays of sub- $100 \mathrm{~nm}$ pitch over large areas is also a challenge due to a strong proximity effect, in addition to the relatively low throughput. Therefore, particular interest for large-area and high-resolution manufacturing has been directed toward EUV lithography, which is considered as the leading candidate for $14 \mathrm{~nm}$ half-pitch processing and below. ${ }^{11,12}$ In parallel with development of projection optical systems for EUV lithography, EUV-IL has been demonstrated to be an effective tool for academic as well as industrial research for fabrication of high-resolution periodic nanostructures down to sub- $10 \mathrm{~nm}$ half-pitch over large-areas with high throughput. ${ }^{11}$ Based on the EUV-IL concept, we recently developed a so-called achromatic spatial frequency multiplication (ASFM) method working in scan-exposure mode. This method is based on the Talbot effect with the use of a broadband light source of limited beam step size, in which the beam is scanned laterally over a large-area mask, enabling averaging of the beam profile over a large area through multiple exposures. With this method, homogeneous one-dimensional (1D) and 2D patterns with sub-50 nm half-pitch were obtained over large areas ${ }^{11,13}$ and with a pattern demagnification factor of 2 relative to the patterns on the mask that is written by EBL. ${ }^{13}$ Using this technique, Si master stamps were fabricated by EUV-IL exposure of resist using Au plated absorber patterns on $\mathrm{Si}_{3} \mathrm{~N}_{4}$ membrane masks and subsequent reactive ion etching (RIE) process at a high throughput [see Figs. 2(a) and 2(b)].

With these Si stamps, NIL was performed according to the process flow described in Fig. 2. To reduce the risk of contamination and damage of the master stamp, working stamps utilized in the thermal NIL were prepared by copying the master stamp using a novel UV-curable organic-inorganic hybrid polymer, i.e., Ormostamp ${ }^{\circledR}[$ Fig. 2(c) $] .{ }^{14}$
Both the original (master) Si stamp and the Ormostamp copies were coated with an antisticking coating, using a mix of two perfluorinated silanes, which was applied from the gas phase in vacuum at room temperature. ${ }^{15}$ For copying, a defined quantity of the viscous Ormostamp precursor was dispensed on a $0.7 \mathrm{~mm}$ thick Borofloat ${ }^{\circledR}$ glass and gently spread after covering with the silicon stamp. ${ }^{14}$ The whole stack was flood exposed to UV light with a dose of about $600 \mathrm{~mJ} / \mathrm{cm}^{2}$ through the transparent glass in an i-line device set-up for $300 \mathrm{~s}$ (ELC-500 UV from Polysciences), followed by a postbake for $30 \mathrm{~min}$ at $130^{\circ} \mathrm{C}$ on a hotplate. After detaching, a first generation hybrid stamp copy with the inverted polarity of the master is obtained. In our experiments, with this stamp thermal NIL patterning of $130-\mu \mathrm{m}$-thick COC foil (Topas, Advanced Polymers $\mathrm{GmbH}$ ) was executed for $10 \mathrm{~min}$ at $120^{\circ} \mathrm{C}$, in a Jenoptik HEX03 thermal precision press. Subsequently, bilayer Al WGPs were realized by directed thermal evaporation of 40-nm thick $\mathrm{Al}$ onto the patterned $\mathrm{COC}$ foil as illustrated in Fig. 2(g).

A $20 \times 20 \mathrm{~mm}^{2} \mathrm{Si}$ master stamp was fabricated by EUV-IL and RIE with the patterned area of $5 \times 5 \mathrm{~mm}^{2}$. Figures 3(a) and 3(b) show SEM of this master stamp (period and depth of $80 \mathrm{~nm}$, linewidth of $32 \mathrm{~nm}$ ). As seen in Fig. 3(b) Si gratings have nearly vertical walls, which were considered to be the optimum structural shape for bilayer WGPs with NIL fabrication. We note that the depth of the etched Si stamp is larger than the designed depth of the polymer grating to be obtained. This is important in order to compensate the depth of the patterned polymer using the two stage NIL process, where the polymer may not completely fill the stamp cavity. For a specific grating period, line width, and NIL process parameters, this reduction in depth was found to be reproducible. ${ }^{4}$ The depth and the width of the final polymer pattern is around $60 \mathrm{~nm}$ and $36 \mathrm{~nm}$, respectively, evaporation of 40-nm-thick $\mathrm{Al}$ yielded the final WGP on COC foil.

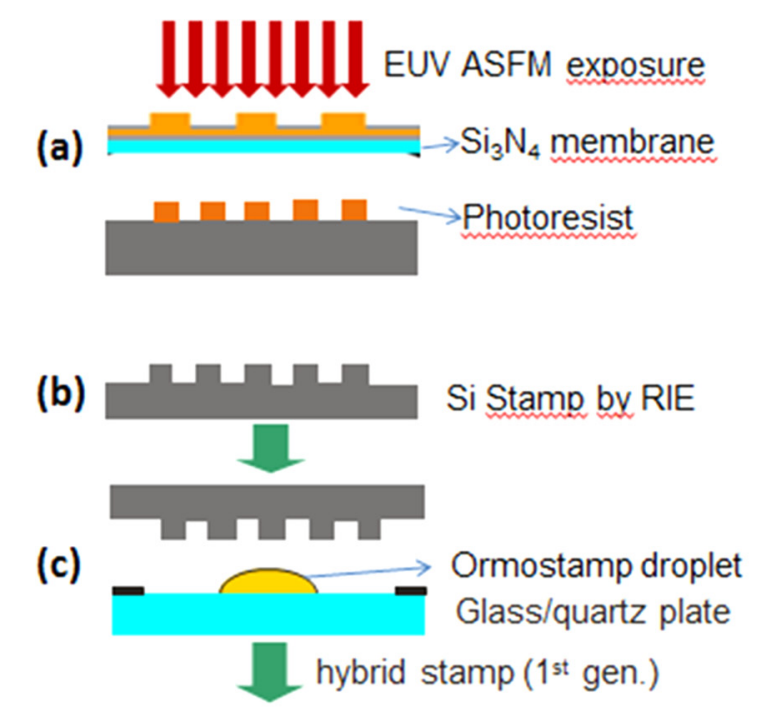

(d)

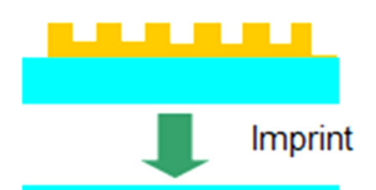

(e)

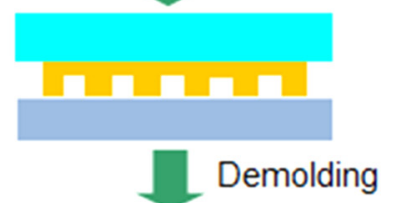

(f)
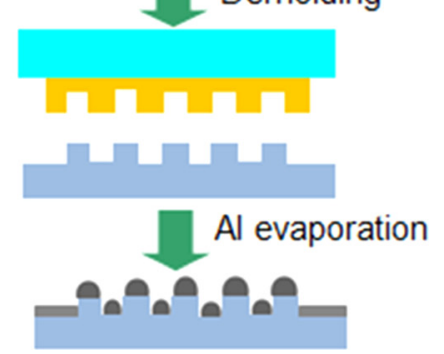

FIG. 2. (Color online) Process flow for fabrication of bilayer WGP on a polymer foil (not to scale). (a) Patterning of photoresist mask using EUV-IL ASFM exposure through a transmission grating with double period absorber pattern. (b) Si master stamp fabrication with EUV-IL exposure and RIE etching of Si. (c) and (d) Fabrication of first generation stamp copy from silicon master. (e) and (f) Thermal imprint into COC foil from first generation stamp. (g) Evaporation of $\mathrm{Al}$ onto the patterned $\mathrm{COC}$ foil to form bilayer WGP. 


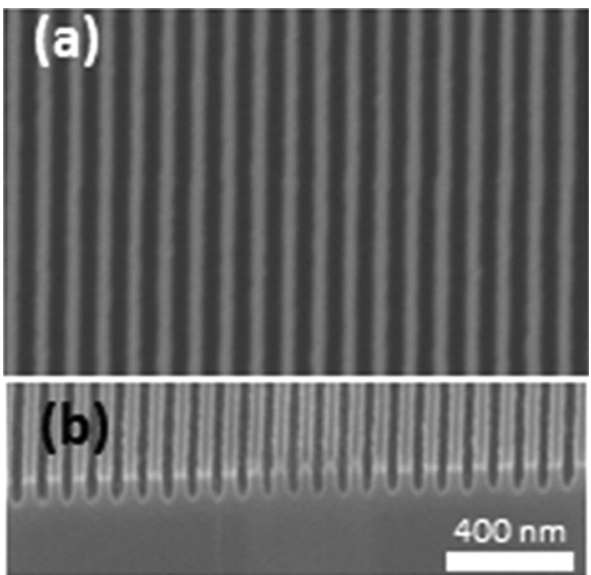

FIG. 3. (a) Top view and (b) cross-sectional SEM images of RIE etched Si master stamp (period $80 \mathrm{~nm}$, linewidth $32 \mathrm{~nm}$, and depth $80 \mathrm{~nm}$ ).

Figures 4(a) and 4(b) show the top view SEM images of patterned COC grating after the fabrication steps of NIL and $\mathrm{Al}$ evaporation, respectively. The linewidth of the top metal grating after evaporation $(48 \mathrm{~nm})$ is larger than the patterned
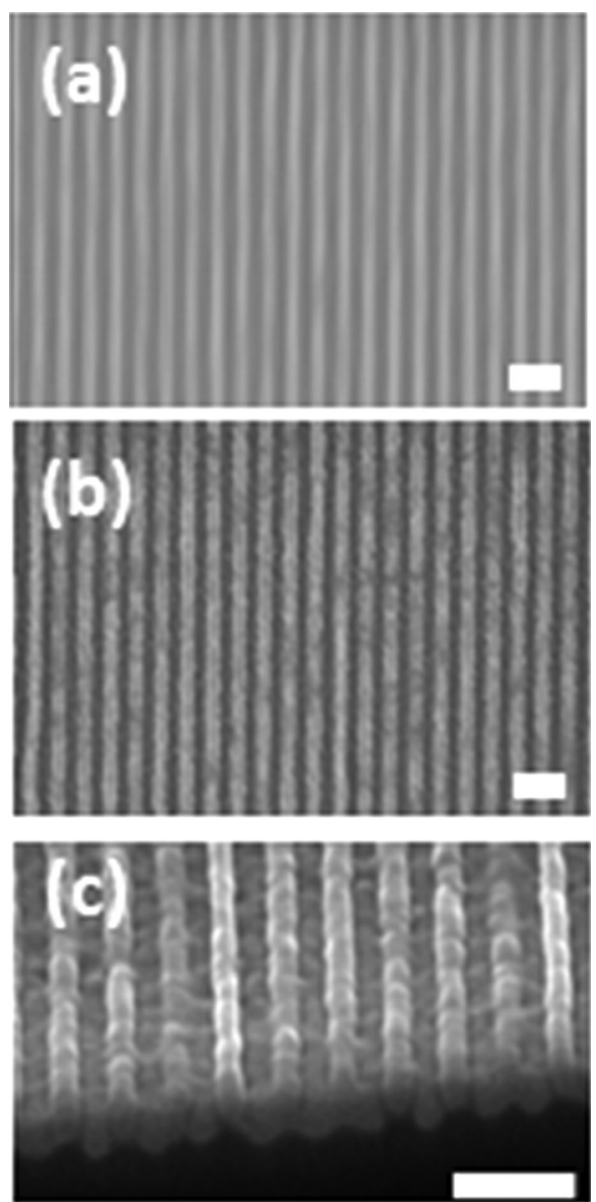

FIG. 4. Top view SEM images of patterned COC polymer grating (period $80 \mathrm{~nm}$ and line width $36 \mathrm{~nm}$ ) and of bilayer WGP: (a) after thermal NIL and (b) after subsequent evaporation of a 40-nm-thick Al layer. (c) Crosssectional SEM image with 40-nm-thick Al layer. All the scale bars are $160 \mathrm{~nm}$. The final polymer structure is probably distorted by FIB milling, since the metal grating is more pronounced that the polymer grating with its shallow sinusoidal appearance.
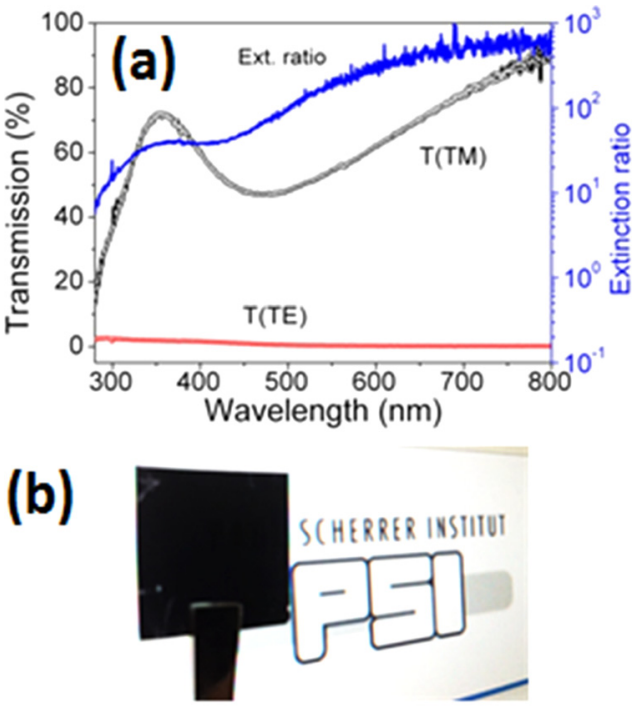

(c)

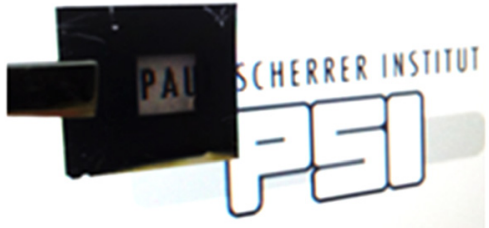

FIG. 5. (Color online) (a) Transmission and extinction ratio spectra of a WGP on a COC foil. (b) and (c) Pictures of a liquid crystal display viewed through the fabricated WGP device working in (b) extinction mode and (c) transmission mode, respectively.

polymer one $(36 \mathrm{~nm})$, as expected because of the fact that during evaporation the top layer gets thicker and the bottom layer gets narrower with increasing thickness due to the shadowing of the deposited layer. For inspection of the cross-sectional profile of evaporated WGP on the polymer foil, focused ion beam (FIB) milling was employed. Imaging of such a cross-section sample by SEM was very challenging because of the nonconductive nature of the polymer substrate. Nevertheless, it can clearly be seen from Fig. 4(c) that two layers of line gratings, separated to each other by a halfpitch shift, were formed.

Optical characterization of the polarizer was done using a Perkin Elmer UV/VIS/NIR two-beam spectrometer in the $280-800 \mathrm{~nm}$ wavelength range with a spectral resolution of $2 \mathrm{~nm}$ at normal incidence. A depolarization unit before the sample chamber and a Glan-Thompson type polarizer after the sample chamber were installed in the spectrometer. Thanks to the large area of the fabricated WGPs, no focusing optics was required. Alignment between the sample axis and the polarization axis of the incoming light was achieved by minimizing the transmission, i.e., alignment of the TE polarization, at a wavelength of $600 \mathrm{~nm}$. A figure with a characteristic maximum (TM polarization) and minimum (TE polarization) of the transmission could be obtained. An accurate measurement of the TE transmission is important for the calculation of the extinction ratio, because minor changes in that value will affect the extinction ratio considerably. Figure 5(a) shows the optical performance of the fabricated WGP on 
the COC foil from the IR region down to the DUV region, where a TM transmission over $70 \%$ and extinction ratio over $30 \mathrm{~dB}$ were achieved. The experimentally obtained TM transmission spectrum agrees very well with the calculated values shown in Fig. 1(b). The measured transmission for TE polarization is two magnitudes higher than in the simulations. We believe that this discrepancy is due to the roughness of the $\mathrm{Al}$ grating wires [see Fig. 4(b)], which cannot be accounted for in the simulations. Nevertheless, the transmission and contrast obtained from the WGPs made by NIL and subsequent metal evaporation are sufficient for various applications. As a visual demonstration of the polarizing properties, one can see that the PSI logo on the liquid crystal display was either opaque or transparent when viewed through the fabricated WGP when the polarization axis of the display is parallel [Fig. 5 (b)] or perpendicular [Fig. 5(c)] to the gratings axis on the WGP, respectively.

\section{CONCLUSIONS}

In conclusion, we have achieved a feasible and costeffective method for the fabrication of high-resolution bilayer WGPs working for a broadband range, from IR down to DUV spectral region. The whole process flow includes four lithography stages, where production costs are reduced through every lithography stage. This is achieved by fabrication of the EUV mask gratings by EBL, from which large area stamp originals are generated using EUV-IL by step and repeat exposure and by multiplying valuable stamp masters using UV-NIL and by high throughput and large area patterning of thermoplastic foils using thermal NIL. All process steps are dedicated to generate or preserve the high aspect nature of the gratings. Optimized geometrical parameters of bilayer WGPs within the feasible range of nanofabrication methods available were obtained by extensive simulations. Based on these results, bilayer Al WGPs with a period of $80 \mathrm{~nm}$ were fabricated. Optical characterizations showed that the fabricated bilayer Al WGPs on a COC foil provide efficient polarizers with a broadband TM transmission of $70 \%$ down to a wavelength of $280 \mathrm{~nm}$, and an extinction ratio of $30 \mathrm{~dB}\left(10^{3}\right)$. In addition, adding an antireflecting layer will improve the transmission as well as protect the Al layer against oxidation. Extinction ratio can be increased by increasing the $\mathrm{Al}$ layer thickness or by improving the deposition conditions to yield $\mathrm{Al}$ wires of reduced roughness. Such Al bilayer WGPs can be used as high-performance, broadband polarizers in applications such as nanophotonics, fiber optics, displays, and detector devices, especially in areas where UV or DUV polarization light is required. Future developments should involve employing advanced roll-to-roll NIL technology as the final stage for further upscaling for patterning of $\mathrm{Al}$ bilayer WGPs that will enable efficient and low-cost polarizers over very large areas, which is required, in particular, for display technology.

\section{ACKNOWLEDGMENTS}

The authors thank Konrad Vogelsang, Michaela Vockenhuber, and Markus Kropf for technical support. This project was funded by the Nano-Argovia programme of the Swiss Nanoscience Institute Basel (SNI). Part of this work was performed at Swiss Light Source (SLS), Paul Scherrer Institute, 5232 Villigen PSI, Switzerland.

${ }^{1}$ S. W. Ahn, K.-D. Lee, J.-S. Kim, S. H. Kim, J.-D. Park, S.-H. Lee, and P.-W. Yoon, Nanotechnology 16, 1874 (2005).

${ }^{2}$ J. J. Wang, F. Walters, X. M. Liu, P. Sciortino, and X. G. Deng, Appl. Phys. Lett. 90, 061104 (2007).

${ }^{3}$ J. J. Wang, W. Zhang, Z. Deng, J. Deng, F. Liu, P. Sciortino, and L. Chen, Opt. Lett. 30, 195 (2005).

${ }^{4}$ H. Schift, J. Vac. Sci. Technol. B 26, 458 (2008).

${ }^{5}$ L. Chen, J. J. Wang, F. Walters, X. Deng, M. Buonanno, S. Tai, and X. Liu, Appl. Phys. Lett. 90, 063111 (2007).

${ }^{6}$ S. H. Ahn, J. S. Kim, and L. Jay Guo, J. Vac. Sci. Technol. B 25, 2388 (2007).

${ }^{7}$ Y. Ekinci, H. H. Solak, C. David, and H. Sigg, Opt. Express 14, 2323 (2006).

${ }^{8}$ S. Park, H. Schift, H. H. Solak, and J. Gobrecht, J. Vac. Sci. Technol. B 22, 3246 (2004).

${ }^{9}$ H. H. Solak and Y. Ekinci, J. Vac. Sci. Technol. B 23, 2705 (2005).

${ }^{10}$ L. Wang, H. H. Solak, and Y. Ekinci, Proc. SPIE 8424, 842429-1 (2012).

${ }^{11}$ L. Wang, H. H. Solak, and Y. Ekinci, Nanotechnology 23, 305303-1 (2012).

${ }^{12}$ C. Wagner and N. Harned, Nat. Photonics 4, 24 (2010).

${ }^{13}$ L. Wang, B. Terhalle, M. Hojeij, V. A. Guzenko, and Y. Ekinci, J. Vac. Sci. Technol. B 30, 031603 (2012).

${ }^{14}$ H. Schift, C. Spreu, M. Saidani, M. Bednarzik, J. Gobrecht, A. Klukowska, F. Reuther, G. Gruetzner, and H. H. Solak, J. Vac. Sci. Technol. B 27, 2846 (2009).

${ }^{15}$ H. Schift, S. Saxer, S. Park, C. Padeste, U. Pieles, and J. Gobrecht, Nanotechnology 16, S171 (2005). 